\title{
Patient identified needs for chronic obstructive pulmonary disease versus billed services for care received
}

\author{
Jill Heins-Nesvold' \\ Angeline Carlson ${ }^{2}$ \\ Leslie King-Schultz ${ }^{3}$ \\ Kenneth E Joslyn ${ }^{4}$ \\ 'American Lung Association \\ of Minnesota, St. Paul, MN, USA; ${ }^{2}$ Data \\ Intelligence Consultants, LLC, Eden \\ Prairie, MN, USA; ${ }^{3}$ Mayo Medical \\ School, Rochester, MN, USA; ${ }^{4}$ Medica \\ Health Plan, Minnetonka, MN, USA
}

Correspondence: Jill Heins-Nesvold American Lung Association of Minnesota, 490 Concordia Avenue, St. Paul,

Minnesota 55103, USA

Tel + I 65I-223-9578

Fax + I 65I-227-5359

Email jill.heins@alamn.org

\begin{abstract}
The American Lung Association of Minnesota (ALAMN) was granted access to a 2004 administrative claims data from an upper mid-Western, independent practice association model health plan. Claims information, including demographics, prevalence, medication and oxygen therapy, and health care utilization, was extracted for 7,782 patients with COPD who were 40 years of age and older. In addition, ALAMN conducted a survey of 1,911 patients from Minnesota diagnosed with COPD. The survey queried the patients about demographics, treatment, medications, limitations, wants, and needs. This article compares and contrasts the information gained through the health plan administrative claims database with the findings from the COPD patient survey in areas of age, gender, types of provider primarily responsible for COPD care, spirometry use, medication therapy, pulmonary rehabilitation, oxygen therapy, and health care utilization. Primary care practitioners provided a majority of the COPD-related care. The claims evidence of spirometry use was $16 \%-62 \%$ of COPD patients had claims evidence of COPD-related medications. $25 \%$ of patients reported, and $23 \%$ of patients had claims evidence of, a hospitalization during the observation year. $16 \%$ of patients reported using pulmonary rehabilitation programs. The results indicate there is an opportunity to improve COPD diagnosis and management.
\end{abstract}

Keywords: chronic obstructive pulmonary disease, oxygen therapy, medication therapy, spirometry, chronic care, assessment

\section{Introduction}

Chronic obstructive pulmonary disease (COPD) is characterized by poorly irreversible airflow obstruction. The airflow limitation usually is progressive and associated with an abnormal inflammatory response related to exposure to noxious substances (GOLD 2006) and is seen most commonly as chronic bronchitis and emphysema. Nearly 10.7 million US adults have been diagnosed with COPD, but as many as 24 million US adults have evidence of impaired lung function (Carlson et al 2006). The disease burden from COPD is marked by significant functional disability, severe respiratory symptoms, and a pronounced impact on quality of life (Vermeire 2002). COPD is the fourth leading cause of death in the United States, claiming the lives of more than 120,000 Americans in 2002 (Carlson et al 2006). In 2004, the economic burden of COPD in the US was estimated at $\$ 37.2$ billion (ALA 2005). Patients with COPD utilize a statistically significantly higher proportion of health care resources, including office visits, inpatient hospitalizations, and prescription drugs than patients who had other nonrespiratory conditions (Shaya et al 2006).

Published estimates of the prevalence of COPD vary greatly. The prevalence of Maryland Medicaid enrollees older than 45 years of age who were continuously enrolled between January 1, 2001 and December 31, 2003 and who had at least one medical claim pertinent to COPD in their primary diagnosis was $1.4 \%$ (Shaya et al 2006). 
An older study conducted in Berlin, New Hampshire reported the prevalences of COPD (defined as forced expiratory volume in the first second $\left[\mathrm{FEV}_{1}\right]<60 \%$ predicted) ranging from $3.1 \%$ to $21.7 \%$ in men in the community where occupational risk factors could have been relevant, and $6.2 \%$ to $13.9 \%$ in women (Ferris and Anderson 1962). In Tecumseh, Michigan, 14\% of adult men and $8 \%$ of adult women had chronic bronchitis, obstructive airway disease, or both (defined as $\mathrm{FEV}_{1}<65 \%$ predicted with an accompanying $\mathrm{FEV}_{1}$ / forced vital capacity [FVC] ratio of 80\%) (Higgins et al 1984). In Glenwood Springs, Colorado, a community screening program found that $17 \%$ of men had chronic bronchitis and 13\% had COPD; $10 \%$ of the women had chronic bronchitis and $4 \%$ had COPD (defined as $\mathrm{FEV}_{1} / \mathrm{FVC}$ ratio, $<60 \%$ with dyspnea, cough, expectoration, or wheeze) (Mueller et al 1971). In the NHANES III study, a national representative sample of adults residing in the US, $8.5 \%$ reported being diagnosed by a physician with obstructive lung disease (Mannino et al 2000).

COPD is also a burden to individuals under 65 years of age who are in the workforce and who are likely to be enrolled in a commercial health plan (Tinkelman et al 2005). In a retrospective analysis of a large dataset of patients with COPD enrolled in a disease management program, almost half $(49.7 \%)$ were less than 65 years of age. In addition, $46.1 \%$ were employed and missed an average of 4.6 days of work the previous six months (Tinkelman and Corsello 2003). Similarly, in a retrospective analysis of a managed care research database, about half of the patients with COPD were in the 45 to 64 year age group (Kesten 2001).

While COPD is the fourth leading cause of death in the United States, there is a lack of data to inform the decisions of the public health, medical, and policy communities (Voekel 2000; Barr et al 2005; Mannino 2005,). The purpose of this study is to assess the concordance of documented health care utilization with patient reported use, needs, and wants regarding COPD in an effort to provide a baseline description of COPD in Minnesota and guide the programmatic efforts of the ALAMN.

\section{Methods}

Two data sources were utilized: the first, a managed care administrative database, and the second, a mailed survey to Minnesota patients identified with COPD.

\section{Administrative data}

This observational cohort study utilized medical and pharmacy administrative claims data from a large, independent practice association model, Midwest health plan (1.25 million insured lives including commercial, Medicaid, and Medicare qualified enrollees). Subjects for this study were identified using medical, pharmacy, and enrollment files. A member, 40 years of age or older during 2004, was included if there was: (1) continuous enrollment from January 1, 2004 to December 31, 2004 with no gaps of enrollment greater than 30 days; (2) there was at least 1 claim with a diagnosis of COPD (491.xx chronic bronchitis; 492.xx emphysema; 496.xx chronic airway obstruction, not elsewhere classified) in any claims diagnosis position; (3) the claim with the associated diagnosis originated from a "medical" place of service (ie, place of service codes at which a patient would likely receive care supervised by a physician).

Variables of interest were determined from the linked data files and included: age, gender, provider with responsibility for COPD care, hospital admissions, emergency department admissions, office visits, drug therapy for COPD (inhaled corticosteroids, anticholinergic agents, bronchodilators (oral or inhaled), combination therapies, oral corticosteroids, theophylline, and cromolyn), spirometry, and oxygen therapy. See Table 1. SAS version 9 (SAS Institute, Cary, NC) was used for all claims analysis.

\section{Patient survey}

\section{Survey development}

Questions from the Seattle Obstructive Lung Disease Questionnaire (Fan et al 2002) were selected based on survey

Table I Coding criteria for COPD claims

\begin{tabular}{ll}
\hline ICD-9 codes & $\begin{array}{l}\text { Medical place of service codes } \\
\text { (US Federal definitions) }\end{array}$ \\
\hline 49I.xx chronic bronchitis & II Office \\
492.xx emphysema & I2 Patient's home \\
496.xx chronic airway & 2 I Inpatient hospital \\
obstruction, not elsewhere & \\
classified & \\
& 23 Emergency room hospital \\
& 26 Military treatment facility \\
Spirometry & 3 I Skilled nursing facility \\
CPT-4 940 I0-94039 & 32 Nursing facility \\
ICD-9-PROC 89.3 & 33 Custodial care facility \\
& 50 Federal qualified health center \\
Oxygen Therapy & 51 Inpatient psychiatric facility \\
HCPCS E0424-E0444; EI390 & 52 Psychiatric facility with partial \\
hospitalization & 53 Community mental health \\
& center \\
56 Psychiatric residential treatment \\
center \\
71 State or local public health clinic \\
72 Rural health clinic
\end{tabular}


length, question content, and psychometrics. ALAMN added additional questions about medication use, physician care, services accessed, barriers to care, and educational or other needs of the patient. Lastly, ALAMN included an analog scale for dyspnea in the past 24 hours.

Experts in lung health research and clinical practice were consulted in the development of this questionnaire. The questionnaire was pilot tested twice in October 2005 with two groups of COPD patients $(n=40)$ who attend ALAMN lung health support groups. Final changes were made to the questionnaire based on feedback from experts and the pilot testing.

\section{Site recruitment and enrollment}

Twelve medical practices across Minnesota were recruited to partner with ALAMN for survey distribution. Sites were selected based on a convenience sample. Clinic sites included both public/academic and private specialty and primary care practices located in the metropolitan area of the Minneapolis/ St. Paul and Greater Minnesota.

Institute Review Board approval was obtained from all institutions requiring it. Each site identified all patients with COPD (defined as ICD-9 code of 491.xx, 492.xx, and 496.xx). Survey packets were prepared at the ALAMN and then transferred to each site for confidential labeling. Sites mailed to all their patients meeting the diagnostic criteria, with the exception of three sites with too large a patient population. In these cases, the total survey number was capped at a random sample of 1000 . The smallest number of surveys mailed to any site was 100 . Surveys were mailed with prepaid return envelope and a $\$ 1$ bill incentive (with exception of one site that did not allow the incentive). In total, 6,860 surveys were mailed; 590 were returned as undeliverable. The usable response rate was $27.9 \%(n=1,911)$. The response rate varied by site, ranging from $8 \%$ to $52.3 \%$.

\section{Data analysis}

Data was hand entered into Remark Office OMR, Version 5.5 (Philadelphia, PN). Data entry validation was conducted on every 50th survey. The accuracy data entry was nearly $100 \%$. Data was analyzed using SPSS version 15.0 for Windows (SPSS Inc., Chicago, IL).

\section{Results}

\section{Demographics}

A total of 7,782 members, 40 years of age or older, were identified with COPD using the health plan claims criteria previously described. Approximately $56 \%$ of members with COPD were female; $78 \%$ were 60 years of age or older. This represents a COPD prevalence rate of 38.3 per 1,000 continuously enrolled members on an annual basis (See Figure 1). While the overall prevalence among women was slightly higher than men (39.5 per 1,000 and 37.0 per 1,000 respectively), this relationship varies by age. At the age of $40-49$, there were statistically significantly more claims for females with COPD than males. At age 60-69, the gender prevalence was similar. In the oldest age group (80 years or older) the prevalence of COPD among continuously enrolled males exceeds the prevalence among continuously enrolled females by a factor of approximately 1.6 times $\left(\mathrm{MH} \mathrm{X}^{2}\right.$ value $\left.=44.91 ; p<0.0001\right)$. Of the 1,911 patients that

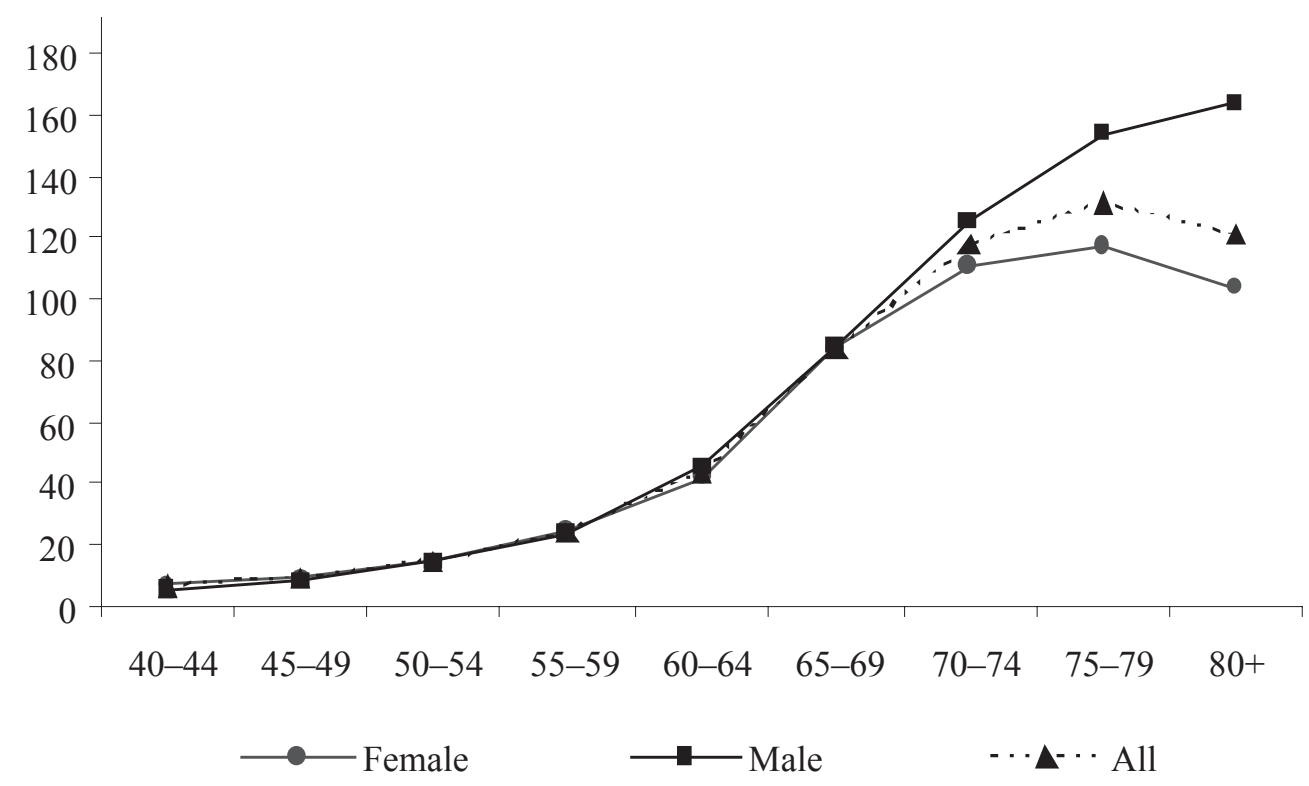

Figure I Prevalence of COPD by age and gender 
completed the Lung Health Questionnaire, 53\% were male and $47 \%$ were female; $82 . \%$ were 60 years of age and older; and $19.1 \%$ were 80 years of age and older.

\section{Provider type responsible for COPD care}

Analysis of claims histories revealed that a family practice provider was identified as responsible for COPD care for $35 \%$ of members identified with COPD; internists for $28 \%$; pulmonary disease specialists for $9 \%$; and $18 \%$ were cared for by a variety of other subspecialists.

Of the patients that completed the Lung Health Questionnaire, 51\% identified a family practice physician as responsible for their COPD care, 13\% identified internists, $27 \%$ identified a pulmonary specialist, and $9 \%$ identified a variety of other health care providers. When asked if the patient had ever seen a lung health specialist or pulmonologist for their breathing problems, $35 \%$ of patients completing the Lung Health Questionnaire indicated no.

\section{Spirometry}

There were 1,258 members with COPD (16\%) who had claims for a spirometry test during the observation year. This is equivalent to one out of every 6 patients with COPD. Of health plan members with COPD and having a claim for a COPD-related medication therapy, $19.4 \%$ had a claim for spirometry.

Of the patients that completed the Lung Health Questionnaire, 60\% (1183) answered that they had at least one breathing test for their lungs ("where you blow hard into a tube, also called spirometry") in the past 12 months.

\section{Drug therapy for treatment of COPD}

Among the 7,782 members identified with COPD, 38\% were without pharmacy claims for a COPD-related drug therapy; bronchodilator (ie, albuterol) use was reported for 38\%; combination therapies (ie, both beta-agonist plus anticholinergic and beta-agonist plus inhaled steroids) for $36 \%$; inhaled corticosteroids (ie, fluticisone and budisinide) for 13\%; anticholinergic agents (ie, ipitrompium) for $14 \%$; oral steroids (ie, prednisone) for 29\%; theophylline for $2 \%$; and cromolyn and nedocromil for $0.1 \%$.

Among patients using inhaled corticosteroids, 6\% had claims evidence of only inhaled corticosteroids. Among the patients using oral steroids, 19\% had claims evidence relying only on these agents. Anticholinergic agents were the only therapy with claims evidence for $5 \%$ of patients with anticholinergic claims. Bronchodilators were the only therapy with claims evidence for $19 \%$ of patients with bronchodilator claims.

Of the patients that completed the Lung Health Questionnaire, the following types of medications were reported as being currently taken for their lung condition: bronchodilators $52 \%$; combination medication $47 \%$; inhaled steroids $30 \%$; anticholinergic agents $27 \%$; prednisone by mouth $12 \%$; and other not specified by patient $20 \%$. Five percent of respondents reported that they did not know the type of medication that they were taking daily for their lung condition.

\section{Health care utilization}

During the observation year, $23 \%$ of the health plan members with COPD had at least one hospitalization for health concerns, whereas $10 \%$ of the general population of continuously enrolled members without COPD had at least one hospitalization. This compares to the $25 \%$ of respondents to the Lung Health Questionnaire that reported they had been hospitalized at least once in the past 12 months.

Utilization of emergency department services, however, was lower among members with COPD than among the general population of continuously enrolled members without COPD (8.4\% vs $13.2 \%$ with at least one ED admission for health concerns). The percentage of COPD members with at least one office visit was also lower than the general population of continuously enrolled members (66\% and $85 \%$, respectively).

Of the 1,991 patients that completed the Lung Health Questionnaire, 39\% reported that they had made at least one unscheduled visit to the doctor's office, urgent care, or the emergency department because of their COPD.

\section{Use of pulmonary rehabilitation}

Pulmonary rehabilitation could not be separately identified in the health plan administrative claims data. Of the patients who completed the Lung Health Questionnaire, however, $2 \%$ reported currently attending a pulmonary rehabilitation program; an additional 1\% were currently in a pulmonary maintenance program; and 13\% have previously attended a pulmonary rehabilitation program. Approximately $66 \%$ of COPD patients who completed the Lung Health Questionnaire reported that they had never been offered or heard of pulmonary rehabilitation programs.

\section{Oxygen therapy}

Claims for any type of oxygen therapy were identified for $22 \%$ of members with COPD. Among these members, $35 \%$ had claims for oxygen concentrators, 32\% for compressed gas, and $33 \%$ for liquid oxygen. 
Of the patients that completed the Lung Health Questionnaire, 31\% reported that they currently use oxygen at home to treat their lung condition. Of those that reported using an oxygen system, $47 \%$ reported using oxygen concentrators; $22 \%$ compressed gas; $18 \%$ liquid oxygen; and $13 \%$ home-fill systems.

\section{Discussion}

The results of the administrative claims study indicate a COPD prevalence rate of 38.3 per 1,000 continuously enrolled members in this independent practice association model health plan. The manner in which prevalence rates increased as aged increased, and were higher among older males than older females, is consistent with published literature. This study's prevalence rate is two times higher than that reported by Shaya and colleagues (2006) among Maryland Medicaid enrollees. In that study, selection criteria included members who were continuously enrolled between January 1, 2001 and December 31, 2003 and who had at least one medical claim pertinent to COPD in the primary diagnosis. However, it is likely this Medicaid only population would have lower prevalence due to the younger average age of Medicaid enrollees.

It is important to note that reported prevalence varies across published studies depending on the claims identification method used. This study used selection criteria of one year of continuous enrollment, and applied ICD-9-CM diagnosis coding from places of service judged to be those where patients would most likely be in contact with a physician. Clearly, a more standardized definition of health plan administrative database selection criteria for patients with COPD is needed.

Approximately $63 \%$ of patients identified in the health plan data were seen by a primary care physician (either a family practitioner or internist) for their COPD care. This was consistent with the $67 \%$ of patients who completed the Lung Health Questionnaire indicating that a generalist (family practice, nurse practitioner, physician assistant, or internist) was primarily responsible for the management of their lung condition.

Only one out of six members with COPD in the administrative data had claims evidence of spirometry during the study year, suggesting that this tool may be underutilized. Literature suggests that underutilization of spirometry in COPD cases may be common. Chapman and colleagues (2001) surveyed 192 primary care physicians using a hypothetical case presentation and structured interview. Only $22 \%$ of the physicians interviewed indicated that they would have requested spirometry after the initial presentation. In an earlier study based on one-on-one interviews with primary care physicians, Kesten and Chapman (1993) concluded that primary care practitioners underutilized spirometry as a screening tool. Access to spirometry, training on implementation and interpretation, and education on its relevance was identified as a major goal for primary care physicians by COPD experts from the United States and Europe (Voelkel 2000).

Sixty percent of respondents to the Lung Health Questionnaire reported having a spirometry test in the past year. This is a frequency much higher than the administrative claims data indicate. The difference in these two results might be explained by either patient perception of a lung test, even though the questionnaire offered a rudimentary definition of spirometry (ie, "Had a breathing test for your lungs where you blow hard into a tube [also called spirometry"]) or the low survey response rate of $28 \%$. In addition, patients who choose to complete the Lung Health Questionnaire might be those who are more aware of their disease and/or are being monitored more carefully by their physician.

Bronchodilators and combination therapies were the most frequently reported medication therapy for both administrative claims data and Lung Health Questionnaire respondents. Bronchodilator therapy is recommended as the first line therapy for all patients with COPD (GOLD 2006). It is of concern that $38 \%$ of patients with COPD did not have claims for any COPD-related drug therapy. While it is true that patients can obtain medications from sources other than insurance (eg, physician samples, out-of-pocket purchases, or mail order outside of the US), given the cost of these medications, it is not likely that patients would choose to bypass their prescription benefit. This finding should not necessarily be interpreted as a failure of the physician to prescribe medications; rather, it may reflect patient choice not to acquire them. Claims data cannot measure prescriptions written and not filled.

Equally concerning, $19 \%$ of patients with COPD only had claims evidence for oral steroids, $6 \%$ for inhaled cortiosteroids only, and 5\% for anticholinergic agents only. These findings are not consistent with national COPD guidelines, which indicate that bronchodilator medications are central to the management of COPD. Bronchodilators should be prescribed on an as-needed or on a regular basis to prevent or reduced symptoms (GOLD 2006).

The results from the administrative claims data indicated that patients with COPD had hospitalizations (25\%) more frequently than they had emergency department visits (8\%). This is consistent with Shaya and colleagues (2006), who found on average 25 claims for inpatient hospitalization over 
a three year period and at most, one claim for an emergency department visit. Emergency department visits for COPD may be low because those COPD patients who present in the emergency department and are subsequently admitted are not reflected in the emergency department rate.

Only 16\% of respondents to the Lung Health Questionnaire have attended or were currently participating in a pulmonary rehabilitation program. Pulmonary rehabilitation has well-documented benefits, such as strength, endurance, and confidence, as well as reducing dyspnea. However, it is underutilized because it is often not reimbursed and is not made available to most COPD patients (Pierson 2004). Data from the Lung Health Questionnaire confirm that pulmonary rehabilitation is not only underutilized, but patients lack awareness about its existence. Nearly two-thirds of the respondents had not heard of pulmonary rehabilitation or had not been offered this as an option by their health care provider.

Twenty-two percent of the patients have claims evidence and $31 \%$ of the respondents to the Lung Health Questionnaire report using oxygen. If guidelines are being followed, this would mean that $22 \%-31 \%$ of these patients have severe COPD, and therefore should be optimally medicated. It would require further study to determine if oxygen therapy was being used appropriately and/or if medication was generally optimal.

Based on these findings, it is clear that there is an opportunity to improve COPD diagnosis and management by focusing on primary care. This focus should include promotion of national care guidelines and professional education related to COPD and respiratory health. A systems approach will be needed to obtain significant improvement in COPD care. The introduction of a chronic care model for COPD may include: accurate patient identification; training of and decision-support tools for health care professionals related to spirometry, COPD diagnosis and appropriate treatment; a multidisciplinary approach to patient care and education; guidelines-based medication and oxygen therapy use; improved clinic flow systems; and provider performance feedback, with appropriate incentives, on process and outcome measures.

\section{Limitations of this study}

Several limitations to this study exist. Claims data limitations include only one year of administrative claims and inclusion of person's with managed care coverage, which does not generally include veterans. There is also a dependence on the accuracy of coding and an individual's choice to utilize health care services for their disease. Lung Health Questionnaire limitations included a $28 \%$ response rate and the inability to test for representatives of the respondents. Authors cannot comment on the severity of COPD disease, the prescribing pattern of physicians, whether patients were appropriately managed by primary care providers, or the need for referrals to specialists.

\section{Acknowledgments}

The authors would like to acknowledge the contributions of Medica Health Plans for the use of their administrative claims data, Boehringer Ingleheim (Ridgefield, Connecticut, United States) for supporting this study, and the 12 clinics who participated in the identification of patients with COPD and distributing the Lung Health Questionnaire. The authors report no conflicts of interest in this work.

\section{References}

Barr RG, Celli BR, Martinez FJ, et al. 2005. Physician and patient perceptions in COPD: The COPD Resource Network Needs Assessment Survey. Am J Med, 118:1514.

Carlson ML, Ivnik MA, Dierkhising RA et al. 2006. A learning needs assessment of patients with COPD. Medsurg Nursing, 15:204-12.

Chapmann KR, Tashkin DP, Pye DJ. 2001. Gender bias in the diagnosis of COPD. Chest, 119:1691-5.

Fan VS, Curtis JR, Tu SP, et al. 2002. Using quality of life to predict hospitalization and mortality in patients with obstructive lung disease. Chest, 122:429-36.

Ferris BG Jr, Anderson DO. 1962. The prevalence of chronic respiratory disease in a New Hampshire town. Am Rev Resp Dis, 86:165-77.

[GOLD] Global Initiative for Chronic Obstructive Lung Disease. 2006. Workshop Report: Global strategy for diagnosis, management, and prevention of COPD [online]. Accessed on January 10, 2008. URL: http://www.goldcopd.com/.

Higgins MW, Keller JB; Landis JR. 1984. Risk of chronic obstructive pulmonary disease: collaborative assessment of the validity of the Tecumseh index of risk. Am Rev Resp Dis, 130:380-5.

Kesten S. 2001. Chronic Obstructive Pulmonary Disease in a managed-care setting. Dis Manage Health Outcomes, 9:589-99.

Kesten S; Chapman KR. 1993. Physician perceptions and management of COPD. Chest, 104:254-8.

Mannino D. 2005. Chronic obstructive pulmonary disease in 2025: where are we headed? Eur Respir J, 26:189.

Mannino DM, Gagnon RC, Petty RL, et al. 2000. Obstructive lung disease and low lung function in adults in the United States: data from the National Health and Nutrition Examination Survey, 1988-1994. Arch Intern Med, 160:1683-9.

Mueller RE, Keble DL, Plummer J. 1971. The prevalence of chronic bronchitis, chronic airway obstruction, and respiratory symptoms in a Colorado city. Am Rev of Resp Dis, 103:209-28.

[NCQA] National Committee for Quality Assurance. 2007. Accessed January 20, 2007. URL: http://www.ncqa.org/programs/hedis/2007/index.htm.

Pierson DJ. 2004. Translating new understanding into better care for the patient with chronic obstructive pulmonary disease. Respir Care, 49:99-109.

Shaya FT, El Khoury AC, Samant ND, et al. 2006. Utilization of health care resources in a high-risk Medicaid population with Chronic Obstructive Pulmonary Disease. Pharm Ther, 31:261-8.

Tinkelman DG, Corsello P. 2003. Chronic Obstructive Pulmonary Disease: the impact occurs earlier than we think. Am J Manag Care, 9:767-71.

Tinkelman DG, George D, Halbert RJ. 2005. Chronic Obstructive Pulmonary Disease in patients under age 65: utilization and costs from a managed care sample. J Occup Environ Med, 47:1125-30.

Vermeire P. 2002. The burden of chronic obstructive pulmonary disease. Resp Med, 96:3-10.

Voelkel NF. 2000. Raising awareness of COPD in primary care. Chest, 117:372-5. 
Appendix A List of lung health questionnaire partner clinics
I. Alexandria Clinic
2. Broadway Clinic (formerly North Memorial Family Practice)
3. Cass Lake Hospital and Clinics
4. Health Partners
5. Immanuel St. Joe's
6. Mayo Clinic
7. Multicare Associates
8. Olmstead County Medical Center
9. St. Luke's Clinic - Duluth
10. St. Mary's Duluth Clinic
II. University of Minnesota
12. Minneapolis Department of Veterans Affairs Medical Center 
